\title{
Maximizing Kolmogorov Complexity for accurate and robust bright field cell segmentation
}

\author{
Hamid Mohamadlou', Joseph C Shope ${ }^{4}$ and Nicholas S Flann 1,2,3*
}

\begin{abstract}
Background: Analysis of cellular processes with microscopic bright field defocused imaging has the advantage of low phototoxicity and minimal sample preparation. However bright field images lack the contrast and nuclei reporting available with florescent approaches and therefore present a challenge to methods that segment and track the live cells. Moreover, such methods must be robust to systemic and random noise, variability in experimental configuration, and the multiple unknowns in the biological system under study.

Results: A new method called maximal-information is introduced that applies a non-parametric information theoretic approach to segment bright field defocused images. The method utilizes a combinatorial optimization strategy to select specific defocused images from each image stack such that set complexity, a Kolmogorov complexity measure, is maximized. Differences among these selected images are then applied to initialize and guide a level set based segmentation algorithm. The performance of the method is compared with a recent approach that uses a fixed defocused image selection strategy over an image data set of embryonic kidney cells (HEK 293T) from multiple experiments. Results demonstrate that the adaptive maximal-information approach significantly improves precision and recall of segmentation over the diversity of data sets.

Conclusions: Integrating combinatorial optimization with non-parametric Kolmogorov complexity has been shown to be effective in extracting information from microscopic bright field defocused images. The approach is application independent and has the potential to be effective in processing a diversity of noisy and redundant high throughput biological data.
\end{abstract}

\section{Background}

Cell segmentation is the identification of cell objects and their observable properties from biological images. Current cell segmentation methods perform most accurately when applied to high contrast and minimal noise images obtained from samples where the cells have fluorescentlylabeled cell nuclei and stained membranes, and are distinct with minimal adherent membranes. However, these ideal conditions rarely exist.

Fluorescently tagging cells using green fluorescent protein (GFP) leads to robust identification of each cell during segmentation. While GFP tagging is widespread, there

\footnotetext{
*Correspondence: Nick.Flann@usu.edu

1 Department of Computer Science, Utah State University, Logan,

UT 84322, USA

2 Institute for Systems Biology, Seattle, WA 98109, USA

Full list of author information is available at the end of the article
}

are disadvantages when applying the method repeatedly to the same sample since under repeated application of high-energy light the cells can suffer phototoxicity. Such light can disrupt the cell behavior through stress, shorten life and potentially confound the experimental results [1-3]. Significantly, a requirement for GFP labeling adds a step before a new cell line can be studied, thus making it difficult to apply this method in a clinical setting.

The alternative is to use bright field microscopy, the original and the simplest microscopy technique, wherein cells are illuminated with white light from below. However, using only bright field imaging of unstained cells presents a challenging cell detection problem because of lack of contrast and difficulty in locating both cell centers and borders, particularly when cells are tightly 
packed. Bright field imaging, while eliminating phototoxicity, leads to an excess of segmentation errors that significantly reduce biological and medical utility.

We seek to remedy the disadvantages and harness the experimental advantages of bright field microscopy of living cells by applying information-theoretic measures over defocused images to improve segmentation accuracy. The approach applies Kolmogorov complexity to identify the most informative subset of images within the focal stack that maximize information content while minimizing the effect of noise.

The paper first briefly reviews existing methods for segmentation of living cells, with a focus on recent approaches to defocused bright field images. Next, measures of Kolmogorov complexity are introduced and applied to image data. The new maximal-information method is then defined and evaluated by comparing its performance with a recent method sephaCe [3] over image sequence data sets from three separate experiments. An analysis and a discussion of the results follows.

\section{Cell segmentation methods}

Several cell segmentation approaches have been developed over time for detection of live cells in microscopy images [4-7]. Most of the approaches binarize an image with certain thresholding techniques, and then use a watershed or level-set based method on either intensity, gradient, shape, differences in individual defocused images (referred to as frames) $[3,8]$, or other measures. The algorithms then remove small artifacts with size filters, and apply merge and split operations to refine the segmentation [4-6].

\section{Florescent microscopy cell segmentation}

Most studies can primarily be categorized into a few key approaches. Wavelets are used for decomposing an image in both the frequency and spatial domain, and can be an effective tool since wavelets are robust to local noise and can discard low frequency objects in the background. Genovesio et al. [9] developed an algorithm to segment cells by combining coefficients at different decomposition levels. Wavelet approaches work well with whole cell segmentation, but have difficulty to segment internal cell structures. In Xiaobo et al. [10] a watershed algorithm was introduced for cell nuclei segmentation and phase identification. Using adaptive thresholding and feature extraction, Harder et al. [11] classified cells into four cell classes comprising of interphase cells, mitotic cells, apoptotic cells, and cells with clustered nuclei. In Solorzano et al. [12] the level set method determines cell boundaries by expanding an active contour around each detected cell nuclei.

While these cell segmentation algorithms have been developed for fluorescence microscopy images, defocused bright field cell segmentation demands more complex and advanced level of image processing. Broken boundaries, poor contrast, partial halos, and overlapping cells are some of the shortcomings of available algorithms $[3,8]$ when applied to images lacking fluorescent reporters.

\section{Defocused bright field microscopy approaches}

Selinummi et al. [13] introduced z-projection based method to replace whole cell florescent microscopy with bright field microscopy. This method computes an intensity variation over a stack of defocused images (referred to as the z-stack) to obtain a contrast-enhanced image called a z-projection. Since variability of pixel intensity inside a cell is high compared to the background, the resulting z-projection image has high contrast and can substitute for an image obtained through whole cell florescent microscopy. The z-projection approach is straightforward and free from parameters setting. However, in order to distinguish between adherent cells, a second channel of nuclei florescent microscopy is required. As a final step CellProfiler [14] software is applied to both the z-projection and nuclei florescent channel to produce cell segmentation. While the $\mathrm{z}$-projection approach avoids whole cell florescence, it still requires an additional nuclei channel of florescent microscopy and so does not eliminate potential problems with cell toxicity.

\section{Implementation}

A recent method that needs only bright-field defocused images has been introduced in sephaCe [3]. This system is capable of both the detection and segmentation of adherent cells and can be downloaded from (http://www. stanford.edu/ rsali/sephace/seg.htm) as a free and open source image analysis package. In contrast to Selinummi et al. where all the frames of the z-stack are utilized, sephaCe selects only a subset of five frames as input to the image processing system. sephaCe selects this subset using a hard-coded strategy independent of each data set and each individual z-stack contained within that data set. Therefore sephaCe does not adapt to the inevitable equipment and biological sample variation. While parameters of the image processing method can be tuned for specific data sets somewhat ameliorating the problem, a more general purpose non-parametric frame selection method is needed for high-throughput processing of diverse data sets. This work introduces a new adaptable frame selection method that applies an information theoretic measure to select frame subsets specific to the idiosyncracies of each z-stack. This method is referred to as maximalinformation.

Following frame subset selection, the maximal information method applies the same image processing and segmentation algorithm of sephaCe. Ali et al. [3,8] presents a series of algorithms that automatically segment each z- 
stack without the need for any florescent channel. The key to discriminating adherent cells is to initialize a level-set algorithm [15] with the difference between two strongly defocused frames and then guide contour expansion using the difference of two weakly defocused frames. As an initial step, the in-focused frame is detected by selecting that image from the z-stack in which the Shannon entropy [16] is minimized. Given an image histogram $I$, entropy is defined as:

$$
\left.E(I)=-\int_{y=1}^{n} \int_{x=1}^{m} p(I(x, y)) \log p(I(x, y))\right) d x d y
$$

Where $p(I(x, y))$ is the probability of pixel intensity values. Entropy value is expected to be maximized for strongly out of focused images and minimized for the in-focus image. Let the in-focus image frame be $I^{0}$.

After detecting the in-focus image, four additional images from the z-stack are selected, two above the infocus frame and two below. To initialize the level set algorithm, a difference image is generated from two strongly defocused images selected at a fixed distance of $\pm 25 \mu \mathrm{m}$ from the in-focus frame, referred to as $I^{++}$and $I^{--}$. This image is binarized using the Otsu [17] thresholding method and then small artifacts are removed by labeling connected components and applying size filter.

To guide the level set algorithm in expanding the initial cell boundaries, another difference image is generated between two slightly defocused images $\pm 10 \mu \mathrm{m}$ from the in-focus frame, referred to as $I^{+}$and $I^{-}$. Details on how this difference image is applied to compute local phase and local orientation images that direct the border expansion is given in [8] and [3].

\section{Motivation for the maximal information approach}

In the sephaCe package the four defocused frames are chosen at fixed distances $( \pm 10 \mu m, \pm 25 \mu m)$ from the in-focused frame to initialize and guide the level-set algorithm. Figure 1(a) illustrates an entropy analysis of a z-stack with 21 frames in which the image separation is $3 \mu \mathrm{m}$. The in focus frame $I^{0}$ is determined as the $12^{\prime}$ th frame, the 9'th and $15^{\prime}$ th frames are the weakly defocused frames $I^{-}$and $I^{+}$(in this case $\pm 9 \mu m$ due to sampling resolution), the strongly defocused frames $I^{--}$and $I^{++}$ are the 4 'th and 20 'th frames. In this z-stack image, as the frames become more blurred, their entropy increases monotonically implying that there are no irregularities within the frames. In this ideal case, the fixed strategy can produce reasonable results.

However, in experiments over a diversity of images (given in Section Results) this fixed selection of out-offocus frames is demonstrated to produce poor segmentation. A fixed strategy cannot take into account random and systemic noise, variability in experimental configurations including microscope configurations, and multiple unknowns in the biological system under study. Some of these conditions are illustrated in selected frame images in Figure 1(c). Two possible reasons to account for the irregular entropy-focus plane relationship in Figure 1(b) are:

- Biological variability where cells do not adhere to the flat surface of the culture medium but vary in the $\mathrm{z}$-dimension as they change morphology and form cell-cell adhesive bonds. That is, a focused frame for one cell could be a defocused frame for other cells. In Figure 1(c), the bright upper cell is positioned higher than the rest. Therefore a semi-random level of sharpness resides in the all defocused images.

- Systemic noise introduced by microscopy and imaging. For instance in Figure 1(c), frame 6 has strip noises introduced by the camera. Strip noise residing in the image increases the entropy value from the 5 th frame to 6 th frame while a decrease is expected.

Applying this fixed distance strategy to select strongly defocused frames can add unwanted initial active contours resulting in over-segmentation and also can miss initial active contours resulting in under-segmentation. Likewise, fixed selection of weakly defocused frames can add anomalies into the local phase and orientation images and thus misdirect the contour expansion to include or exclude cells, particularly when cells are tightly packed.

Overall, the fixed approach in selecting initial images in the sephaCe package is brittle and error-prone. The unavoidable variation requires an adaptable method rather than a fixed approach. The maximal-information method uses an optimization based approach that searches the combinations of z-stack frames to select the four frames that contain the highest information, evaluated using Kolmogorov information-theoretic measure [18]. This process is repeated for each individual z-stack and so adapts to the distinctiveness of each sample. Since the maximal-information method is adaptive, it can be applied to a diversity of data sets utilizing different microscopes, lighting conditions and biological samples.

\section{Kolmogorov information set complexity}

Set complexity [19], denoted $\Psi$, is applied to quantify the amount of information contained within each possible set of four image frames. The measure is general purpose and non-parametric in that it computes the information content of set of objects so long as they can be encoded as strings. Set complexity has been applied to understand the organization and information content of biological data sets including developmental pattern formation [20], 

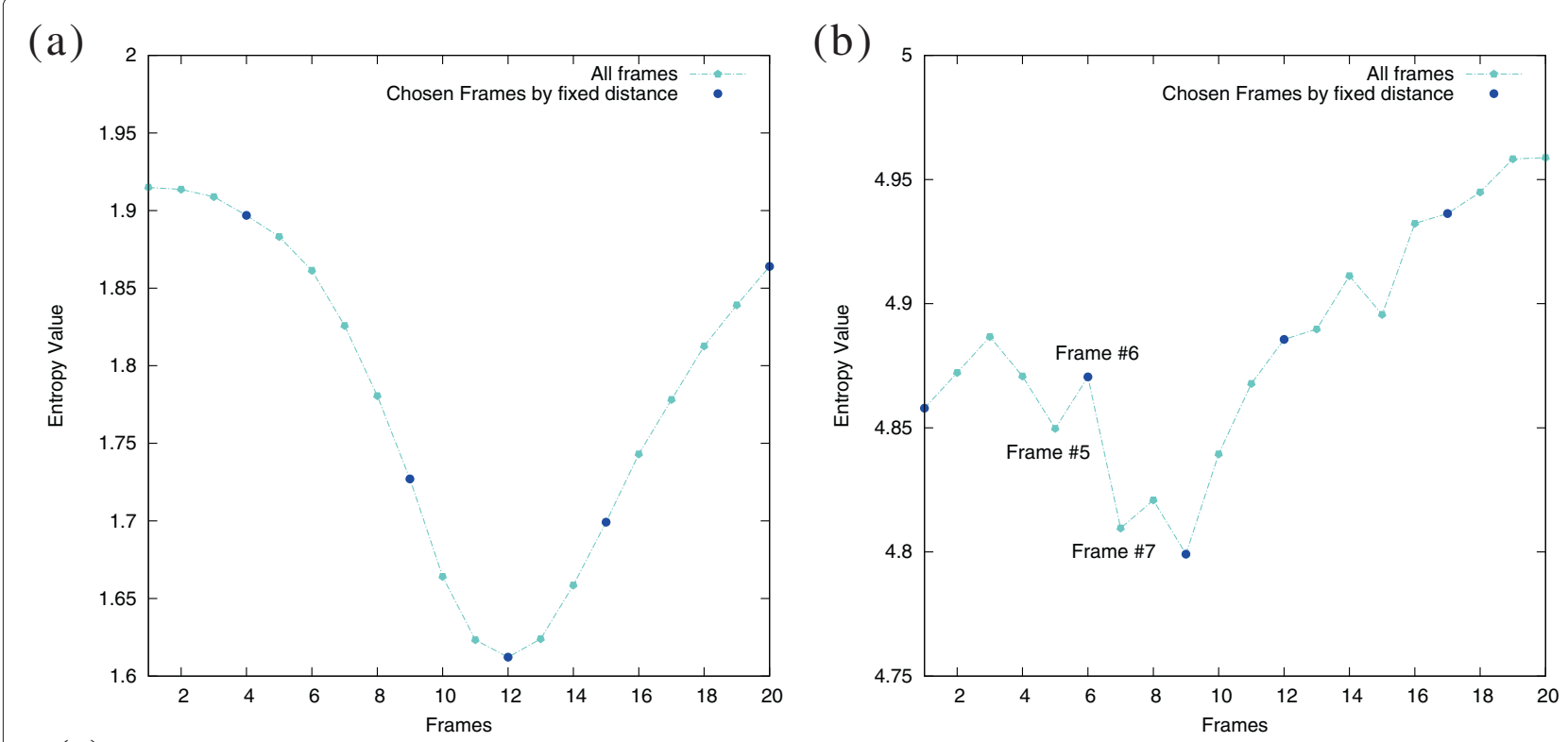

(c)

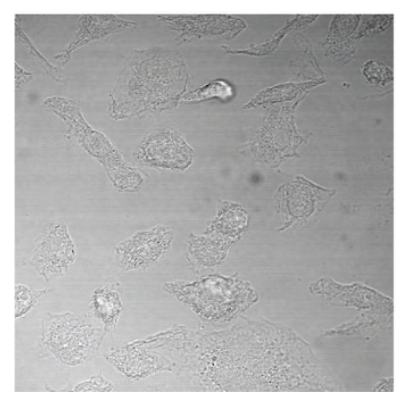

Frame \#4

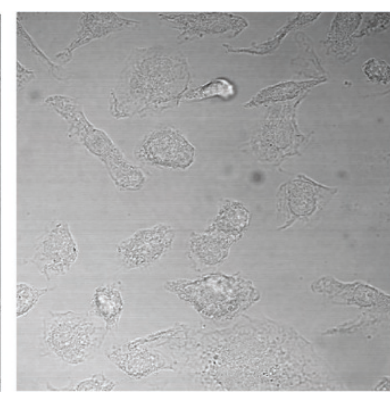

Frame \#5

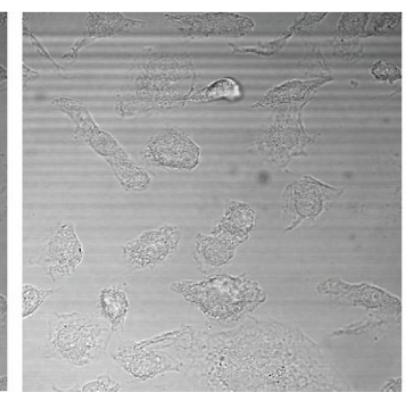

Frame \#6

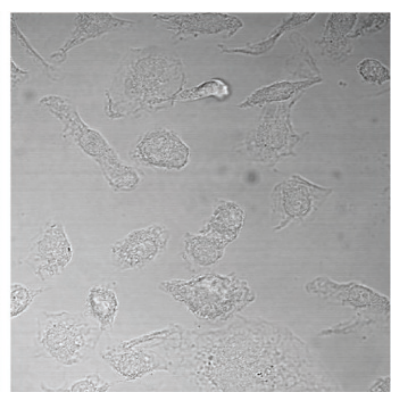

Frame \#7

Figure 1 Relationship between frame entropy as the focus level changes in the $\mathbf{z}$-stack is shown in (a) and (b). In (a) there is a monotonic increasing and then decreasing relationship between focus and entropy, with the in-focus frame containing minimum entropy. In (b) a nosier data set is employed and the relationship between focus and entropy is irregular. As can be seen in frame 6 , banding and stripe noise introduced by the microscope unexpectedly increases entropy. (c) Illustrates four corresponding frames for data set analyzed in graph (b).

genetic regulatory network dynamics [21], and gene interaction network structure [22]. The Kolmogorov complexity [18] of a string is the length of shortest algorithm that can be used to generate the string. Exact computation is undecidable, but it can be approximated by the compression size of a string. Bzip2 and zip compressor with block size of 900 Kbytes have been tested and shown robust for this purpose.

A related Kolmogorov complexity measure is the Normalized Compression Distance NCD) defined as the length of the shortest program that computes one given string from another. This measure provides a quantification of similarity between the strings since the more similar they are, the shorter the program needed. Again, this measure is undecidable but can be estimated using compression. Normalized Compression Distance described in [23] and [24] defined below, is such a measure of similarity between two objects that applies compression size $C(s)$ of string $s$ :

$$
N C D\left(s_{i}, s_{j}\right)=\frac{C\left(s_{i}+s_{j}\right)-\min \left(C\left(s_{i}\right), C\left(s_{j}\right)\right)}{\max \left(C\left(s_{i}\right), C\left(s_{j}\right)\right)}
$$

Where $s_{i}+s_{j}$ is the concatenation of $s_{i}$ and $s_{j}$ string. If the two strings compress smaller together than separately, then $N C D$ will be closer to 0.0. As the two strings are more similar, the concatenated string is more compressed resulting in a lower $N C D$ value. Random strings or dissimilar regular patterns are not as compressed and so $N C D$ will be closer to $1[25,26]$.

1. $C\left(s_{i}^{s}+s_{j}^{s}\right) \simeq C\left(s_{i}^{s}\right) \simeq C\left(s_{j}^{s}\right)$ then $N C D\left(s_{i}^{s}, s_{j}^{s}\right) \simeq 0.0$

2. $C\left(s_{i}^{r}+s_{j}^{r}\right) \simeq C\left(s_{i}^{r}\right)+C\left(s_{j}^{r}\right)$ then $N C D\left(s_{i}^{r}, s_{j}^{r}\right) \simeq 1.0$

3. $C\left(s_{i}^{r}+s_{j}^{s}\right) \simeq C\left(s_{i}^{r}\right)$ and $C\left(s_{j}^{s}\right) \simeq 0.0$ then $\operatorname{NCD}\left(s_{i}^{r}, s_{j}^{s}\right) \simeq 1.0$ 
Where $s^{r}$ is from the set of random strings and $s^{s}$ are simple strings containing regular patterns.

Set complexity [19] of a set of $n$ strings $S=\left\{s_{1}, \ldots, s_{n}\right\}$ is defined:

$$
\Psi(S)=\frac{1}{n(n-1)} \sum_{s_{i} \in S} C\left(s_{i}\right) \sum_{s_{j} \neq s_{i}} N C D\left(s_{i}, s_{j}\right)\left(1-N C D\left(s_{i}, s_{j}\right)\right)
$$

Set complexity captures the relationships among strings in the set, discounting when strings are very similar $(N C D$ close to 0.0 ) and so contain the same information, or highly dissimilar so that they have nothing in common and appear random (NCD closer to 1.0). The value is maximized when each string is intrinsically complex (high $C\left(S_{i}\right)$ ) and the similarity between the strings lies between maximally dissimilar and maximally similar $N C D\left(s_{i}, s_{j}\right) \simeq$ 0.5 , which occurs when $C\left(s_{i}+s_{j}\right) \simeq C\left(s_{i}\right) / 2-C\left(s_{j}\right)$, assuming $C\left(s_{i}\right)>C\left(s_{j}\right)$.

Figure 2 gives an example of applying $\Psi(S)$ to defocused images. Along the top are the original frames and below them is their binary representation following an Otsu thresholding step. Each binary image is encoded as a string by concatenating each column scanning from left to right (more details are provided in Algorithm 1). For each image the compression size is given. NCD values between each pair of the images is provided in Table 1.

\section{The maximal-information segmentation method}

To select the four most informative frames from a z-stack with $n$ frames, the method searches the space of all possible combinations of two frames from above the in-focus frame $\left(I^{++}\right.$and $\left.I^{+}\right)$and two frames from below the infocus frame $\left(I^{-}\right.$and $\left.I^{--}\right)$, evaluates each set for $\Psi$, then picks the maximizing combination. The method is given in Algorithm 1.

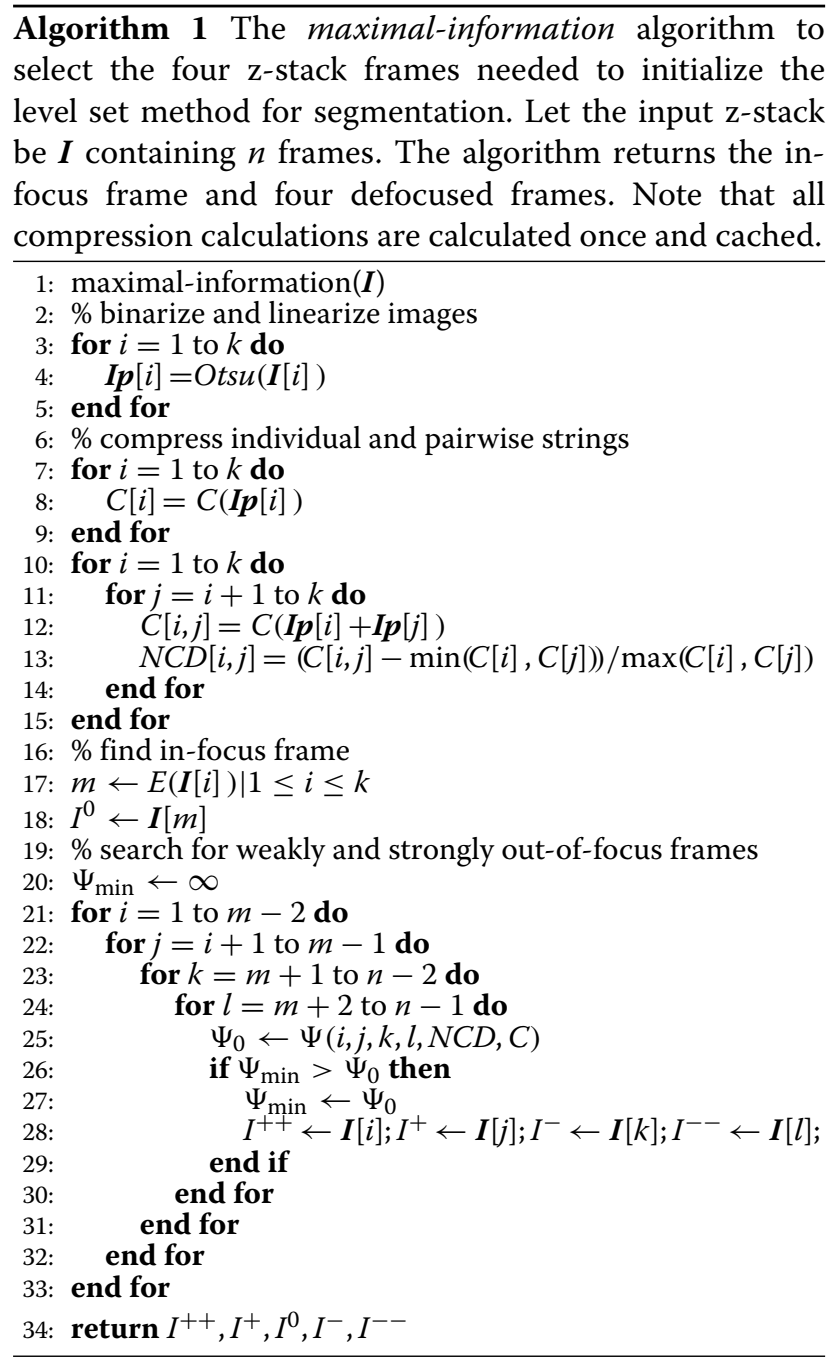

First each image in the z-stack is binarized using the Otsu [17] thresholding method and then converted to a string (linearization) by concatenating each column of the image to the next column [27]. Many methods of linearization were explored in [27] and column

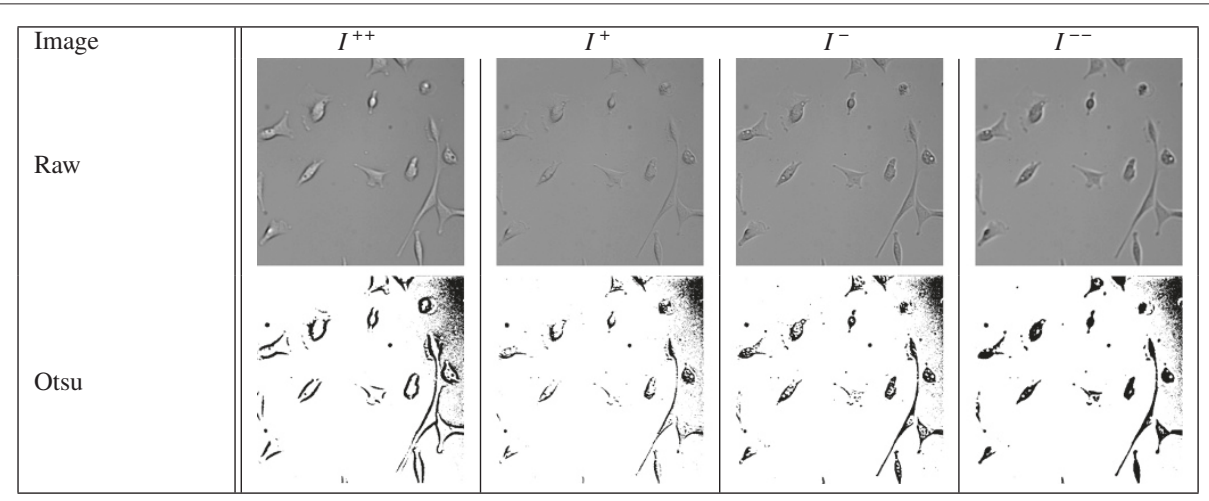

Figure 2 Strongly and weakly defocused selected frames from time step 1 in data set one. Top row is the raw image frames. The second row is the binary image following Otsu thresholding that is linearized and compressed. 
Table 1 The NCD values for the four image frames given in Figure 2

\begin{tabular}{ccccc}
\hline NCD & $\boldsymbol{I}^{++}$ & $\boldsymbol{I}^{+}$ & $\boldsymbol{I}^{-}$ & $\boldsymbol{I}^{--}$ \\
\hline$I^{++}$ & 0.0 & 0.1429 & 0.2154 & 0.1071 \\
$I^{+}$ & 0.0 & 0.0 & 0.2615 & 0.1296 \\
$I^{-}$ & 0.0 & 0.0 & 0.0 & 0.2000 \\
$I^{--}$ & 0.0 & 0.0 & 0.0 & 0.0 \\
\hline
\end{tabular}

concatenation was found to be effective because spatially located regularities are picked up by compression. Bzip2 is applied to compute the compression size of each individual string and also each pairwise concatenated string (for NCD, Equation 2). From these cached compression values, pairwise $N C D$ values are determined.

The $O\left(n^{2}\right)$ compression step dominates the computation time since strings must be written to file before processing; the final $\Psi$ calculation involves only matrix operations and is very fast, even though more combinations must be computed. For the three data sets studied in this work, the preprocessing and level set algorithms of sephaCe take approximately 10 seconds per z-stack. The maximal-information frame selection method adds approximately 20 seconds per z-stack to the run time. Timings were on an Intel Pentium G640 Processor 2.8 $\mathrm{GHz}$ (3 MB cache).

\section{Results}

\section{Set complexity analysis of image data}

To understand how Kolmogorov Complexity measures could reveal information in z-stacks, an initial study was performed by computing the NCD between each pair of 21 frames for three data sets each containing 192 z-stacks. The data sets used for in this work are human embryonic kidney cells (HEK 293T) sampled at 5 minute intervals for 16 hours. Each $\mathrm{z}$-stack sequence is from a distinct experiment. Data was obtained using a Leica DM6000 microscope with each z-stack containing 21 image frames each separated by $10 \mu m$, with resolution $1024 \times 1024$ 12-bit grey-scale pixels. Since the z-stack was sampled at a $10 \mu \mathrm{m}$ resolution, the strongly defocused frames for sephaCe were set at $\pm 30 \mu \mathrm{m}$.

Figure 3 presents values of $N C D$ in the form of a heatmap for each pair of frames along the z-stack sequence for a selection of three images. Frames tend to decrease in similarity as the focus distance increases so that blue areas (low NCD) are mostly around the diagonal, and red areas off the diagonal. However, each image displays significant individuality due to noise, microscope variability over time and changes in the biological sample as cells divide, die and move. This inconsistency among $N C D$ matrices over time justifies the need for an adaptive frame selection strategy.

Four frames of the z-stack are chosen to start and guide the level set algorithm. Figure 4 compares the computed $\Psi$ of frames obtained by the maximal-information method with the $\Psi$ of the frames identified using the fixed distance method of sephaCe, for all $192 \mathrm{z}$-stacks. In all cases the maximal-information frame set has a higher information content then the fixed sephaCe set. While this result is not surprising, it supports the need for adaptability as it demonstrates the inability of a fixed strategy to pick those images that have high intrinsic information. A mean difference hypothesis statistical analysis demonstrates that these differences are significant, see Table 2. According to the p-value in Table 2, that is much lower than 0.05 , the mean difference hypothesis is rejected and so there is a significant difference between the mean values of the two groups. That is, selecting images using maximal-information guarantees sets with higher $\Psi$ than the sephaCe method.

\section{Precision and recall analysis}

Two examples of segmented bright field microscopy frames are shown in Figure 5. In (a) both algorithms select
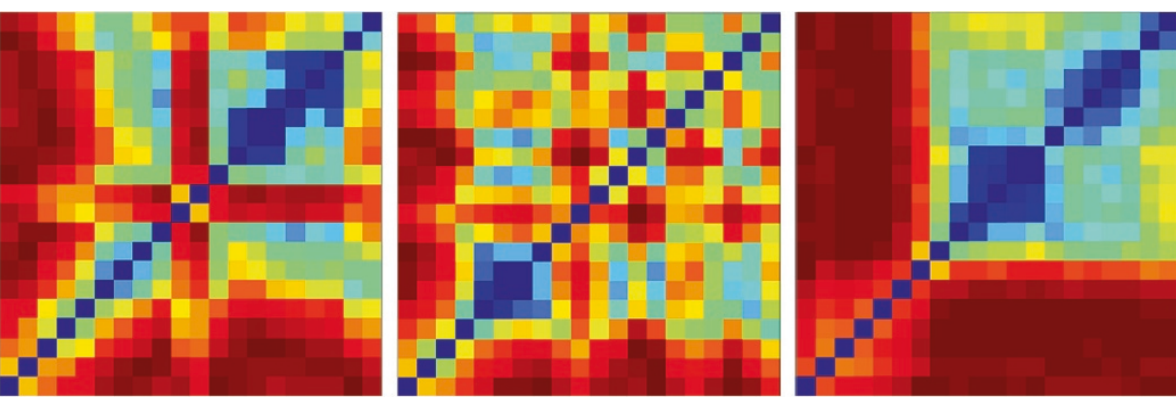

Figure $3 N C D$ values shown as a heatmap for all pairs of image frames in the z-stack of three selected defocused image stacks from the same experiment. Color code blue specifies pairs of frames with lowest NCD values and red specifies highest NCD values. In each heatmap, the lowest $z$ frame is in the lower left, the highest $z$ frame is in the upper right. Analysis illustrates that off-diagonal NCD values range from 0.6 (most similar images) to 1 (red, most dissimilar images). Along the diagonal NCD equals zero (blue). Note the diversity of similarity relationships among the frames of each z-stack. 


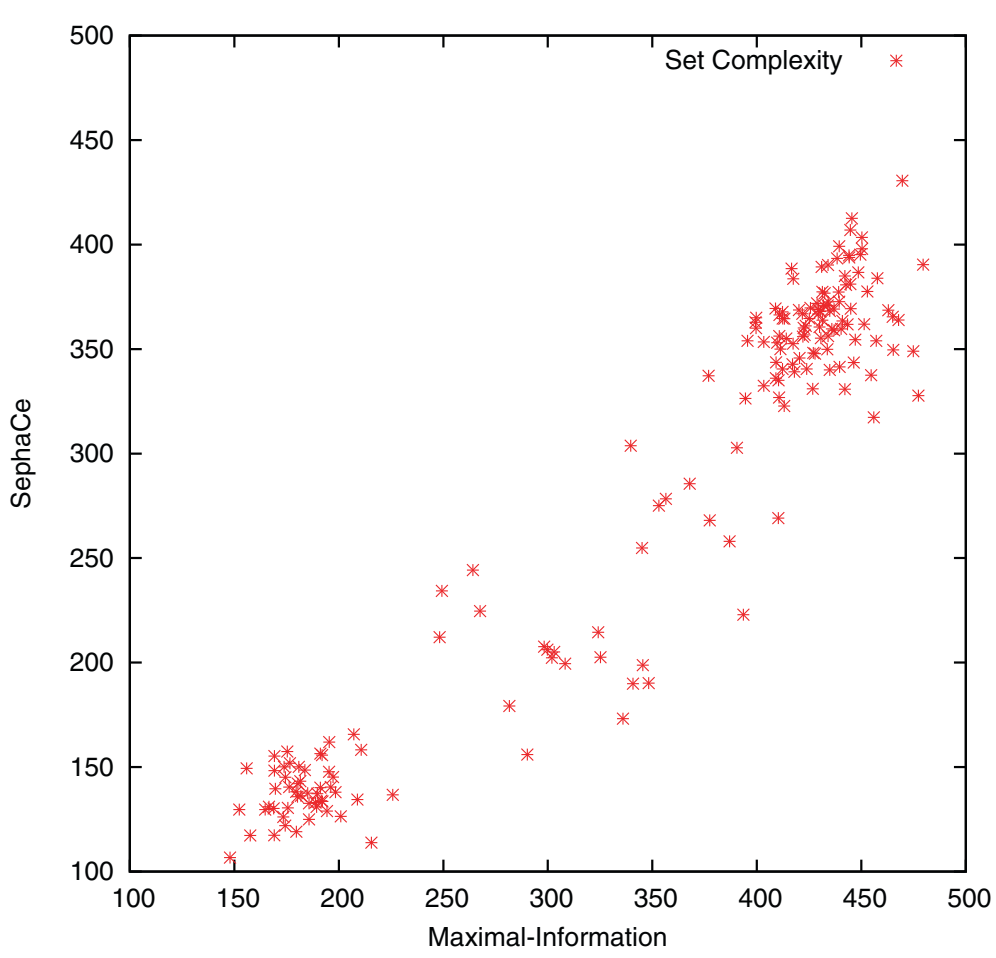

Figure 4 A parametric plot of set complexity values for the four defocused frames selected by the two algorithms. The $X$ axis indicates the complexity value of the frame set selected by maximal-information and the $Y$ axis indicates complexity value for the frame set selected by sephaCe. Each data point represents one z-stack from the 192 z-stacks in the human embryonic kidney cells (HEK 293T) data set.

similar frames and produce similar and accurate results. In (b) maximal-information selects a alternative set of frames at different focus planes (compared to the fixed strategy) and produces significantly lower segmentation errors. Here the sephaCe method fails to accurately detect four cells along with over-segmenting another.

In order to evaluate the segmentation results, the raw microscope z-stacks were provided to a human expert (Joseph C. Shope, Utah State University) who identified the cells using Image-Pro Plus (Media Cybernetics). Optimal $\mathrm{z}$-frames were selected and cell centers determined

Table 2 Set complexity values for two different approaches

\begin{tabular}{ccc}
\hline & $\begin{array}{c}\text { Fixed defocused } \\
\text { distance (sephaCe) }\end{array}$ & $\begin{array}{c}\text { Selected by } \\
\text { maximal-information }\end{array}$ \\
\hline Mean & 278.5049 & 345.1289 \\
Variance & 10620.73 & 12336.47 \\
Observations & 192 & 192 \\
Pearson correlation & 0.9603 & \\
$\mathrm{P}(\mathrm{T}<=\mathrm{t})$ one-tail & $1.19825 \mathrm{E}-67$ & \\
$\mathrm{t}$ Critical one-tail & 1.6536 & \\
$\mathrm{P}(\mathrm{T}<=\mathrm{t})$ two-tail & $2.3965 \mathrm{E}-67$ & \\
$\mathrm{t}$ Critical two-tail & 1.9736 & \\
\hline
\end{tabular}

by fitting a major and minor axis to produced excel files of cell center coordinates for each z-stack. No segmentation results were given to the expert during this initial cell identification. In parallel, the two methods were applied to the data sets to produce segmentation results for each zstack, drawn as overlays with red (maximal-information) and blue (sephaCe) as in Figure 5. Next, the segmentation results were overlaid with the expert-determined cell centers and for both methods a count was made of the correctly identified cells (true positive), missing (false negative) and fragments of cells identified as one cell or spurious objects (false positive). To measure the quality and utility of the methods overall, the precision $\mathrm{Pr}$ and recall Re of maximal-information and sephaCe correction was determined, where:

$$
\operatorname{Pr}=\frac{t p}{t p+f p} \quad R e=\frac{t p}{t p+f n}
$$

with $t p, f p, f n$ being the count of detected true positive, false positive, and false negative objects, respectively. In Table 3 the precision and recall of maximal-information are both significantly better than sephaCe for each of the three data sets.

In Table 3 the average correctly segmented cells for maximal-Information is higher than sephaCe method and 
(a)

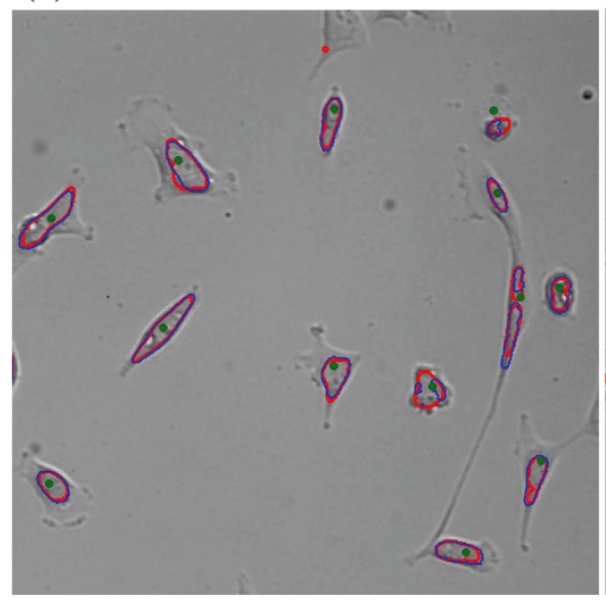

(c)
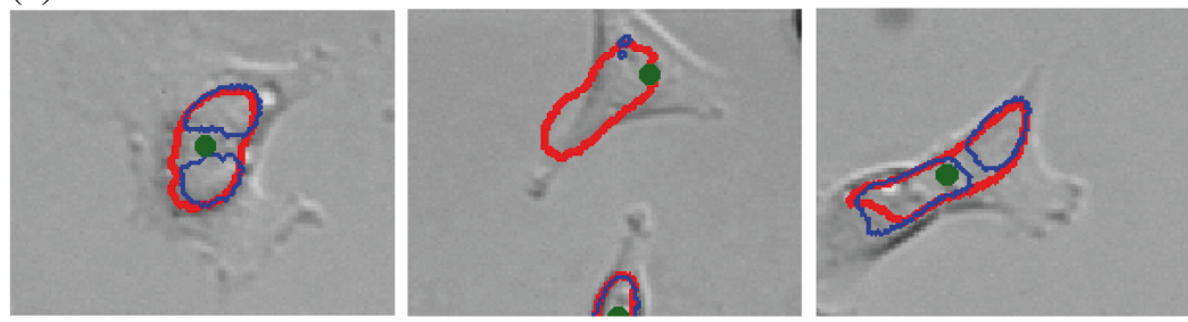

Figure 5 Example cell segmentation results for two z-stacks of human embryonic kidney cells (HEK 293T) overlaid on the in-focus frame. Segmentations produced by maximal-information are shown in red; segmentations produced by sephaCe are shown in blue. In (a) both algorithms select similar frames and produce similar and accurate results. In (b) maximal-information selects a alternative set of frames at different focus planes from the fixed strategy and produces significantly lower segmentation errors. Here the sephaCe method fails to accurately detect four cells along with over segmenting another. In (c) segmentation results are shown closeup.

Table 3 Segmentation results for three data sets for human embryonic kidney cells (HEK 293T)

\begin{tabular}{|c|c|c|c|c|c|}
\hline Data set one & Maximal-information & SephaCe & Correlation & t- stat & $P(T \leq t)$ one-tail \\
\hline Correct segmentation tp & 9.12 & 5.76 & 0.3970 & 9.4557 & 0.0 \\
\hline Unexpected areas $f p$ & 0.68 & 0.80 & 0.2355 & -0.5492 & 0.2939 \\
\hline Missing cells fn & 1.60 & 4.72 & -0.0909 & -9.0929 & 0.0 \\
\hline Precision $\operatorname{Pr}$ & $93.20 \%$ & $89.36 \%$ & 0.3295 & 1.4461 & 0.0805 \\
\hline Recall Re & $85.37 \%$ & $54.34 \%$ & -0.2903 & 8.2830 & 0.0 \\
\hline Data set Two & Maximal-information & SephaCe & Correlation & t stat & $P(T \leq t)$ one-tail \\
\hline Correct segmentation tp & 13.35 & 12.60 & 0.4344 & 3.4701 & 0.0012 \\
\hline Unexpected areas $f p$ & 1.15 & 2.20 & 0.1633 & -4.0977 & 0.0003 \\
\hline Missing cells fn & 0.50 & 1.25 & 0.2939 & -3.4701 & 0.0012 \\
\hline Precision $\operatorname{Pr}$ & $92.30 \%$ & $85.45 \%$ & 0.1690 & 4.3714 & 0.0001 \\
\hline Recall Re & $96.40 \%$ & $91.08 \%$ & 0.2822 & 3.4407 & 0.0013 \\
\hline Data set three & Maximal-information & SephaCe & Correlation & t stat & $P(T \leq t)$ one-tail \\
\hline Correct segmentation tp & 15.56 & 11.86 & 0.4549 & 10.18 & 0.0 \\
\hline Unexpected areas $f p$ & 1.72 & 2.00 & 0.3642 & -0.9434 & 0.1759 \\
\hline Missing cells $f n$ & 2.81 & 6.36 & 0.4926 & -9.9501 & 0.0 \\
\hline Precision $\operatorname{Pr}$ & $91.66 \%$ & $86.23 \%$ & 0.3887 & 2.6898 & 0.0 \\
\hline Recall Re & $85.94 \%$ & $65.21 \%$ & 0.4256 & 10.12 & 0.0 \\
\hline
\end{tabular}

$t p$ is the average count of correctly identified cells, $f p$ is unexpected segmentations and $f n$ is cells that were missed. Recall and precision are given as percentages. 
demonstrates the advantage of extracting more informative frames in the $\mathrm{z}$-stack. The average of both missing and unexpected cell segmentation for maximal-information are lower than sephaCe method. All three of these measures of quality are shown to be significantly better for maximal-information than for the sephaCe using a paired one-tail T-test (values that are less than $10^{-8}$ are reported as 0.0 in the table).

In addition, Table 3 includes the inter-method correlation of $t p, f p, f n$ over the z-stack data sets. High correlation implies that the performance of both methods is consistent in that they perform poorly on the same set of "difficult" images, and well on the same set of "easy" images. Results in Table 3 show that true positives are highly correlated implying that the cells correctly identified by maximal-information include some of the set of cells recognized by sephaCe.

\section{Conclusions}

This work has presented a method for identifying live cells in bright field defocused images. The method applies Kolmogorov complexity measures to identify specific outof-focus frames that encode the maximum information. These frames are then used to initialize active contours and guide contour expansion for level-set segmentation algorithms as applied in the sephaCe method.

The new maximal-information approach is compared with a selection strategy employed in the original sephaCe that picks out-of-focus frames using fixed offsets from the estimated in-focus frame. An empirical study using a large data set of embryonic kidney cells (HEK 293T) z-stacks taken from different experimental runs has demonstrated that the adaptive method significantly improves the recall and precision of the segmentation.

Kolmogorov set complexity identifies the most informative frames by exploiting similarity measures between all pairs of frames contained within the NCD matrix. Each selected frame is sufficiently dissimilar (high $N C D$ ) to other frames in the set so as to provide unique and synergistic information about each cell in the z-stack. Recall that the dissimilarity is due to changes in cell appearance as the focal plane is moved through the cell profile. By selecting the best degree of dissimilarity, the differences between frames (used to initialize and guide the active contour of the level-set method) maximize sensitivity to the presence and shape of cells. Kolmogorov set complexity also tempers the effects of noise by discounting frames that have too higher dissimilarity since this is most likely due to noise.

The method introduced here is generally applicable because it relies on fundamental non-parametric information-theoretic properties and treats data as simple strings, ignoring the actual semantics. Robustness is achieved by viewing frame selection as combinatorial optimization problem with set complexity as the scoring function. The full potential of the method in dealing with noise, variability in experimental configurations, and multiple unknowns across a diversity of biological data will be explored in further studies.

\section{Availability and requirements}

Project name: maximal-information

Project home page: https://sites.google.com/site/maxi malinformation,

Operating system(s): Platform independent

Programming language: Matlab

Other requirements: requires sephaCe [3] downloaded from (http://www.stanford.edu/ rsali/sephace/seg.htm License: GNU GPL

Any restrictions to use by non-academics: Contact corresponding author

\section{Competing interests}

The authors declare that they have no competing interests.

\section{Authors' contributions}

$\mathrm{HM}$ and NSF conceived the method and wrote the manuscript. HM wrote the code and performed all the computational experiments. JS assisted with the writing, analyzed all the raw images and evaluated performance. All the authors have read the paper and approve its contents.

\section{Acknowledgements}

This work was supported by the Luxembourg Centre for Systems Biomedicine, the University of Luxembourg and the Institute for Systems Biology, Seattle, USA. Research reported in this publication was partially supported by the National Institute Of General Medical Sciences of the National Institutes of Health under Award Number P50GM076547. The content is solely the responsibility of the authors and does not necessarily represent the official views of the National Institutes of Health. Thanks to Ilya Shmulevich and Pekka Ruusuvuori for helpful discussions, and to Adrian Ozinsky for image data.

\section{Author details}

${ }^{1}$ Department of Computer Science, Utah State University, Logan,

UT 84322, USA. ${ }^{2}$ Institute for Systems Biology, Seattle, WA 98109, USA.

${ }^{3}$ Synthetic Biomanufacturing Institute, Logan, UT 84322, USA. ${ }^{4}$ Department of Biology, Utah State University, Logan, UT 84322, USA.

Received: 19 March 2013 Accepted: 18 December 2013

Published: 30 January 2014

References

1. Folkard M, Prise KM, Grime G, Kirkby K, Vojnovic B: The use of microbeams to investigate radiation damage in living cells. App/Radiat Isot 2010, 67(3):436-439.

2. Selinummi J, Ruusuvuori P, Podolsky I, Ozinsky A, Gold E, Yli-Harja O, Aderem A, Shmulevich I: Bright field microscopy as an alternative to whole cell fluorescence in automated analysis of macrophage images. PLOS ONE 2009, 4(10):e7497+. [http://dx.doi.org/10.1371/journal. pone.0007497]

3. Ali R, Gooding M, Szilágyi T, Vojnovic B, Christlieb M, Brady M: Automatic segmentation of adherent biological cell boundaries and nuclei from brightfield microscopy images. Mach Vision Appl, 2011:1-15. [http://dx.doi.org/10.1007/s00138-011-0337-9]

4. Adiga PSU, Chaudhuri BB: An efficient method based on watershed and rule-based merging for segmentation of 3-D histo-pathological images. Pattern Recognit 34 2001, 34:1449-1458.

5. Lin G, Chawla MK, Olson K, Guzowski JF, Barnes C a, Roysam B: Hierarchical, model-based merging of multiple fragments for improved three-dimensional segmentation of nuclei. J Cytometry 2005, 63:20-33. 
6. Chen X, Zhou X, Wong STC: Automated segmentation, classification, and tracking of.pdf. IEEE Trans Biomed Eng 2006, 53(4):762-766. doi:10.1109/TBME.2006.870201.

7. Harder N, Neumann B, Held M, Liebel U, Erfle H, Ellenberg J, Eils R, Rohr K Automated recognition of mitotic patterns in fluorescence microscopy images of human cells. In 3rd IEEE International Symposium on Biomedical Imaging: Nano to Macro; 2006:1016-1019. doi:10.1109/ISBI.2006.1625093.

8. Ali R, Gooding M, Christlieb M, Brady M, Feng FRS: Advanced phase-based segmentation of multiple cells from brightfield microscopy images. In 5th IEEE International Symposium on Biomedical Imaging; 2008:181-184. doi:10.1109/ISBI.2008.4540962.

9. Genovesio A, Liedl T, Emiliani V, Parak WJ, Coppey-Moisan M, Olivo-Marin $\mathrm{JC}$ : Multiple particle tracking in 3-D+t microscopy: method and application to the tracking of endocytosed quantum dots. IEEE Trans Image Process: Publ IEEE Signal Process Soc 2006, 15(5):1062-1070.

10. Zhou X, Li F, Yan J, Wong STC: A novel cell segmentation method and cell phase identification using Markov Model. IEEE Trans Inf Technol Biomed 2010, 13(2):152-157.

11. Kovalev V, Harder N, Neumann B, Held M, Liebel U, Erfle H, Ellenberg J, Eils $\mathrm{R}$, Rohr K: Feature selection for evaluating fluorescence microscopy images in genome-wide cell screens. 2006 IEEE Comput Soc Conf Comput Vis Pattern Recognit 2006, 1:276-283.

12. De Solorzano CO, Malladi R, Lelièvre S a, Lockett SJ: Segmentation of nuclei and cells using membrane related protein markers. J Microscopy 2001, 201(Pt 3):404-415.

13. Selinummi J, Ruusuvuori P, Podolsky I, Ozinsky A, Gold E, Yli-Harja O, Aderem A, Shmulevich I: Bright field microscopy as an alternative to whole cell fluorescence in automated analysis of macrophage images. PloS one 2009, 4(10):e7497.

14. Carpenter A E, Jones TR, Lamprecht MR, Clarke C, Kang IH, Friman O, Guertin D a, Chang JH, Lindquist R a, Moffat J, Golland P, Sabatini DM: CellProfiler: image analysis software for identifying and quantifying cell phenotypes. Genome Bio/ 2006, 7(10):R100.

15. Gooding MJ, Kennedy S, Noble JA: Volume segmentation and reconstruction from freehand three-dimensional ultrasound data with application to ovarian follicle measurement. Ultrasound Med Biol 2008, 34(2):183-195.

16. Shannon CE: A mathematical theory of communication. SIGMOBILE Mob Comput Commun Rev 2001, 5:3-55. [http://dx.doi.org/10.1145/ 584091.584093]

17. Otsu N: A threshold selection method from gray-level histograms. IEEE Trans Syst 1979, C:62-66.

18. Andrey N, Kolmogorov: Three approaches to the quantitative definition of information. Problems Inf Transm 1965, 1:1-7.

19. Galas DJ, Nykter M, Carter GW, Price ND, Shmulevich I, Member S: Biological information as set-based complexity. 2010, 56(2):667-677.

20. Flann NS, Mohamadlou H, Podgorski GJ: Kolmogorov complexity of epithelial pattern formation: the role of regulatory network configuration. Biosystems 2013, 112(2):131-138. [http://dx.doi.org/10. 1016/j.biosystems.2013.03.005]

21. Mäki-Marttunen T, Kesseli J, Kauffman S, Yli-Harja O, Nykter M: Of the complexity of Boolean network state trajectories. In Proceedings of the Eighth International Workshop on Computational Systems Biology, WCSB 2011, June 6-8. Zurich, Switzerland. TICSP series.

22. Sakhanenko NA, Galas DJ: Complexity of networks I: The set-complexity of binary graphs. Complexity 2011, 17(2):51-64. [http://dx.doi.org/10.1002/cplx.20382]

23. Chen X, Francia B, Li M, Mckinnon B, Seker A: Shared information and program plagiarism detection. In IEEE Transactions on Information Theory; 2004:1545-1551. [http://citeseerx.ist.psu.edu/viewdoc/summary? doi=10.1.1.10.76]

24. Cilibrasi R, Vitanyi PMB: Clustering by compression. IEEE Trans Inf Theory 2005, 51(4):1523-1545. [http://dx.doi.org/10.1109/TIT.2005.844059]

25. Li M, Li X, Li X, Ma B, Vitanyi P: The similarity metric. In IEEE Transactions on Information Theory; 2003:863-872. [http://citeseerx.ist.psu.edu/ viewdoc/summary?doi=10.1.1.58.1259]

26. Cebrián MC, Alfonseca M, Ortega A: Common pitfalls using normalized compression distance: what to watch out for in a compressor. Commun Inf Syst 2005, 5:367-384. [http://citeseerx.ist.psu.edu/viewdoc/ summary?doi=10.1.1.104.9265]
27. Mortensen J, Wu J, Furst J, Rogers J, Raicu D: Effect of image linearization on normalized compression distance. In Signal Processing, Image Processing and Pattern Recognition, Volume 61 of Communications in Computer and Information Science. Edited by Ślezak D, Pal S, Kang BH, Gu J, Kuroda H, Kim T h. Berlin, Heidelberg: Springer; 2009:106-116. [http://dx.doi.org/10.1007/978-3-642-10546-3_14]

doi:10.1186/1471-2105-15-32

Cite this article as: Mohamadlou et al:: Maximizing Kolmogorov Complexity for accurate and robust bright field cell segmentation. BMC Bioinformatics 2014 15:32.
Submit your next manuscript to BioMed Central and take full advantage of:

- Convenient online submission

- Thorough peer review

- No space constraints or color figure charges

- Immediate publication on acceptance

- Inclusion in PubMed, CAS, Scopus and Google Scholar

- Research which is freely available for redistribution

Submit your manuscript at www.biomedcentral.com/submit
Ciomed Central 